\title{
Sevoflurane anesthesia in electroconvulsive therapy: a meta-analysis of randomized controlled trials
}

\author{
Yoshiteru Takekita ${ }^{1}$, Taro Suwa ${ }^{2}$, Hirotsugu Kawashima ${ }^{3}$, Nobuatsu Aoki ${ }^{1}$, Masaki Kato ${ }^{1}$, \\ Toshihiko Kinoshita ${ }^{1}$
}

${ }^{I}$ Department of Neuropsychiatry, Kansai medical university, Japan, ${ }^{2}$ Department of Neuropsychiatry, Graduate School of Medicine, Kyoto University, Japan, ${ }^{3}$ Department of Psychiatry, Toyooka Hospital, Japan

Sevoflurane is an inhalation anesthetic frequently used in general surgery. In recent years, a few clinical trials have also examined the effects of using sevoflurane in electroconvulsive therapy (ECT). The objective of this study was to provide an up-to-date and comprehensive review on how the sevoflurane anesthesia affects seizure duration and circulatory dynamics, quality of seizure in ECT. We performed a meta-analysis of RCTs that investigated seizure duration and circulatory dynamics, quality of seizure in patients treated with ECT using sevoflurane (sevoflurane group) and other anesthetics (others group). A total of 11 RCTs (364 patients and 1343 ECT sessions) were included. The sevoflurane group showed a significantly shortened seizure duration during ECT compared to the others group [motor: 9 studies, $\mathrm{SMD}=-0.55,95 \% \mathrm{CI}(-1.05,-0.06), \mathrm{p}=0.03$; electroencephalogram: 8 studies, SMD $=-0.85,95 \% \mathrm{C}(-1.23,-0.47), \mathrm{p}$ $<0.00001$. The heart rate was significantly increased in the sevoflurane group compared to the other group [8 studies, $\mathrm{SMD}=0.36,95 \% \mathrm{CI}(0.13,0.58), \mathrm{p}=0.002]$. There were no significant differences in the mean artery pressure or postictal suppression index between the two groups [mean artery pressure: 6 studies, SMD $=3.15,95 \%$ CI $(-4.62$, -10.92), $\mathrm{p}=0.43$; postictal suppression index: 3 studies, $\mathrm{SMD}=0.12,95 \% \mathrm{C}(-0.14,0.38)]$. These results suggest that, in ECT, sevoflurane can be used for patients in whom intravenous anesthesia is not feasible, although particular attention to circulatory dynamics is required. 